\title{
ESTRATEGIAS DOCENTES PARA LA ADAPTACIÓN DE LA DOCENCIA EN CIENCIAS JURÍDICAS AL ENTORNO ON LINE Y SEMIPRESENCIAL
}

Educational strategies for the adaptation of legal science teaching to the on line and blended learning environment

Recibido: 11 de mayo de 2021

Aceptado: 7 de julio de 2021

Raúl Ruiz Rodríguez (coord.) Investigador predoctoral (FPU 2019) r.ruiz@ua.es Universidad de Alicante

Carolina Soler García Prof. Ayudante Doctora carolina.soler@ua.es Universidad de Alicante

Carmen María García Mirete Prof. Contratada Doctora carmen.garcia@ua.es Universidad de Alicante

José Miguel Beltrán Castellanos Prof. Ayudante Doctor jmiguel.beltran@ua.es Universidad de Alicante

Francisco José Abellán Contreras Prof. Ayudante fj.abellan@ua.es Universidad de Alicante
Lerdys Heredia Sánchez Prof. Ayudante Iheredia@umh.es Universidad Miguel Hernández

Alfonso Ortega Giménez Profesor Titular alfonso.ortega@umh.es Universidad Miguel Hernández

Alicia Fernández-Peinado Martínez Prof. Contratada Doctora alicia.fernandez@ua.es Universidad de Alicante

Aitana Ramón Martín Prof. Asociada aitana.ramon@ua.es Universidad de Alicante

Tamara Funes Beltrán Prof. Ayudante tamara.funes@ua.es Universidad de Alicante

\section{RESUMEN}

La situación sanitaria provocada por la COVID-19 ha forzado a las universidades españolas a adoptar herramientas y medidas orientadas a una adaptación de la enseñanza e-learning. La docencia en materias jurídicas partía de una situación inicial de desventaja al hacer uso habitualmente de metodologías de carácter tradicional. Para solventar este problema, un grupo de profesoras y profesores de la UA y la UMH ha diseñado e implementado una serie de estrategias

\section{PALABRAS CLAVE}

docencia virtual, docencia semipresencial, ciencias jurídicas, estrategias, metodologías. 
y metodologías docentes con el fin de adaptar la enseñanza al entorno virtual de manera satisfactoria. El presente trabajo recoge y expone las experiencias docentes desplegadas durante el curso académico 2020/2021 en determinadas asignaturas del ámbito jurídico. Del análisis de estas experiencias se deriva que el catálogo actual de herramientas tecnológicas $y$ metodologías docentes permite alcanzar el citado objetivo de adaptación al entorno virtual. No obstante, existen una serie de factores que escapan al control del profesorado y que requieren el compromiso del resto de miembros de la comunidad universitaria.

\begin{abstract}
the health situation caused by COVID-19 has forced Spanish universities to adopt tools and measures aimed at adapting e-learning education. The teaching of legal subjects was initially at a disadvantage, as it usually made use of traditional methodologies. To solve this problem, a group of lecturers from the UA and the UMH have designed and implemented a series of strategies and methodologies in order to adapt teaching to the virtual environment in a satisfactory manner. The present work gathers and exposes the experiences deployed during the academic year 2020/2021 in certain subjects in the legal field. The analysis of these experiences shows that the current catalogue of technological tools and methodologies makes it possible to achieve the aforementioned objective of adapting to the virtual environment. However, there are a series of factors beyond the control of the teaching staff and which require the commitment of the rest of the members of the university community.
\end{abstract}

\section{KEYWORDS}

virtual teaching, blended learning, legal sciences, strategies, methodologies. 
Sumario: 1. Introducción. 2. Experiencias docentes. 2.1. Derecho Administrativo. 2.2. Derecho Internacional Público. 2.3. Cooperación jurídica internacional. 2.4. Nacionalidad y Extranjería. 2.5. Derecho Penal Especial. 2.6. Historia del Derecho y de las Instituciones. 2.7. Derecho Procesal. 2.8. Derecho internacional privado. 2.9. Derecho del Trabajo. 3. Valoración de los resultados. 4. Conclusiones. 5. Bibliografía.

\section{Introducción.}

La situación sanitaria actual provocada por la COVID-19 ha forzado a las universidades españolas a adoptar herramientas y medidas orientadas a una adaptación de la enseñanza e-learning. Además, ha sido necesario implementar metodologías que permitieran mantener cierto grado de normalidad en el desarrollo de los estudios de grado y máster, dentro del clima de incertidumbre y cambio constante al que los miembros de la comunidad universitaria se encuentran sometidos (Tripathi \& Amann, 2020).

Por este motivo y con la necesidad de lograr una docencia que cumpla con los contenidos de las correspondientes asignaturas en este nuevo contexto, el profesorado universitario ha tenido que reformular la metodología empleada hasta el momento. Y todo ello para facilitar al alumnado la transición al nuevo modelo de docencia on line I semipresencial y asegurar que los objetivos planteados en la guía docente (desarrollo de determinadas competencias, logro de los resultados de aprendizaje previstos, etc.) son alcanzados.

En este sentido, la enseñanza de las disciplinas jurídicas parte de una situación inicial de desventaja. Como se ha señalado, las ciencias jurídicas se han caracterizado normalmente por el uso de metodologías docentes de carácter tradicional (Bastante \& Moreno, 2019: 30). Ejemplo de ello sería la clase magistral, en la que el alumnado se limita a recibir información transmitida oralmente por el profesorado, participando de manera ocasional en el desarrollo activo de la clase. Esta técnica docente sigue empleándose frecuentemente en la enseñanza del Derecho a día de hoy (Elgueta \& Palma, 2014: 917). Es cierto que las citadas metodologías tradicionales han sido eventualmente renovadas mediante un uso limitado de las TICs; por ejemplo, a través de la incorporación de presentaciones de diapositivas o formularios on line. No obstante, su idoneidad en las enseñanzas jurídicas en línea queda en entredicho (Letelier, 2016: 129). Esto es así, en tanto que la existencia de un mayor número de distracciones en el entorno virtual (circunstancias personales y familiares de cada estudiante, multitud de factores que pueden entorpecer la atención del estudiante, entre otras) dificulta el seguimiento de la clase por parte del alumnado (33de Carvalho, 2017: 117). Dicha situación se acentúa cuando no hay una participación activa de los discentes, ni se hace uso de recursos o metodologías innovadoras capaces de atraer su atención. A ello hay que sumarle un menor grado de motivación provocado por factores tales como el empeoramiento en el estado de salud mental derivado de la pandemia (Cobo-Rendón et al., 2020; Moya-Lacasa et al., 2021) o el cambio constante de criterios de asistencia y presencialidad ${ }^{1}$.

Con el fin de tratar de salvar todos los obstáculos aquí descritos, un grupo de profesoras y profesores de las Facultades de Derecho de la Universidad de Alicante (en adelante, "UA") y de la Universidad Miguel Hernández (en adelante, "UMH") propuso, al inicio del curso académico 2020-2021, una planificación ordenada y estructurada de las correspondientes asignaturas jurídicas que permitiera hacer uso de nuevas metodologías y/o herramientas virtuales adecuadas al nuevo contexto de enseñanza-aprendizaje. Este

\footnotetext{
${ }^{1}$ Ver en: https://elpais.com/educacion/2020-11-01/la-universidad-se-juega-el-desencanto-y-abandono-desus-nuevos-alumnos.html, visitado el día 15 de septiembre de 2021.
} 
grupo de profesores ha tratado de adaptar los contenidos y metodologías a este nuevo escenario e-learning, compartiendo sus diferentes estrategias docentes, para así tratar de alcanzar conclusiones y resultados en común. La finalidad es contribuir a mejorar el proceso de enseñanza-aprendizaje en las ciencias jurídicas, a partir de fórmulas adaptadas a un entorno digital que contribuyan a la formación y a la asunción de competencias de los estudiantes.

En suma, el presente trabajo está dedicado a analizar y valorar las experiencias docentes y los resultados obtenidos a partir de la implementación de las distintas metodologías $e$ learning en las enseñanzas jurídicas. Todo ello, teniendo en cuenta las particularidades que revisten cada una de las materias impartidas, así como sus necesidades concretas y las principales dificultades que entraña su implementación.

\section{Experiencias docentes.}

\subsection{Derecho Administrativo}

El modelo de docencia dual impuesto en la UA por la situación de emergencia sanitaria no supone un cambio sustancial con respecto a la docencia tradicional o presencial, sino solo un cambio en el canal de comunicación. Dicho de otro modo, supone que las sesiones docentes se imparten utilizando una herramienta distinta, aunque bien es cierto que la relación de cercanía, colaboración, sensibilidad y respeto mutuo se torna más gélida en ausencia del contacto físico, lo que puede influir en la baja intervención del alumnado (Maré \& Teedzwi, 2021:165). No obstante, del mismo modo que, por ejemplo, el uso de medios electrónicos en las Administraciones Públicas no supone un cambio en los aspectos sustantivos de los procedimientos administrativos, sino solo la forma (instrumentos) a través de la cual se materializan (principalmente sedes y registros electrónicos), la docencia dual mantiene, con toda lógica, los mismos objetivos y competencias que el alumnado deberá adquirir, si bien con ciertas peculiaridades que traen causa de no estar físicamente en el aula durante buena parte del curso.

En este contexto, el uso de la tecnología debe buscar acercar lo máximo posible una experiencia docente plena al alumnado que, por no corresponderle el turno de presencialidad o por no poder asistir al aula por causas justificadas (confinamiento perimetral, cuarentena, persona de riesgo) seguirá la sesión a través de la aplicación informática habilitada por la Universidad. Entre las fortalezas que se les atribuyen a las tecnologías digitales en este ámbito, Martínez et al. (2016:289) subrayan su capacidad para alcanzar a un mayor número de personas, optimizar la comunicación y romper las barreras espacio-temporales, favoreciendo el desarrollo de una atención más personalizada. En esta misma línea, Maré \& Teedzwi (2021:166) sugieren que esta modalidad permite crear un ambiente positivo e impulsar la creación de comunidades de aprendizaje que disminuyen la sensación de aislamiento. Ahora bien, el cambio de modalidad de presencial a on line o semipresencial puede suponer una barrera para adquirir los conocimientos, y es respecto de esta cuestión cuando se requiere aplicar estrategias docentes para compensar las eventuales dificultades que puedan surgir.

En concreto, las estrategias docentes implementadas en las asignaturas de Derecho Administrativo I y II del Grado en Derecho de la Universidad de Alicante se han basado en mejorar la transmisión de conocimientos y el refuerzo del aprendizaje, al tiempo que se ha centrado la preocupación por el buen funcionamiento de la sesión on line y el mantenimiento de la atención del alumnado.

De este modo, ante la falta de poder utilizar con relativa solvencia la pizarra tradicional, o el hecho de que se pierda buena parte del lenguaje no verbal (gestos con las manos, por 
ejemplo) se ha apostado por otras herramientas audiovisuales que suplan estas carencias, y que permitan al alumnado identificar cuáles son los contenidos más importantes o aquellas cuestiones clave que les permitirán superar con éxito la asignatura, de modo que como señala Álvarez-Rosa et al. (2018:50) la expansión de las tecnologías de la información y la comunicación (TIC) ha supuesto "un cambio metodológico en los procesos de enseñanzaaprendizaje de todas las esferas educativas". Por tanto, esta situación ha comportado un cambio progresivo en la metodología docente aplicada en las aulas universitarias, ya que la metodología tradicional (la lección magistral referenciada en la Introducción) se ha venido complementando con otros métodos docentes más innovadores mediante la utilización de recursos tecnológicos (Soto, 2020:195).

En este sentido, se ha recurrido a la elaboración de presentaciones PowerPoint que, si bien no son nuevas en el ámbito docente, han cobrado una mayor virtualidad por facilitar el impacto visual de las explicaciones durante las sesiones on line. En efecto, las posibles dificultades que pueden surgir para escuchar al profesorado durante las clases, por ejemplo, por mala calidad del audio o interrupciones puntuales de la conexión a la red, quedan en buena parte compensadas si el alumnado recibe el contenido también por escrito, de forma esquematizada y clara en dichas presentaciones.

Ahora bien, dichas diapositivas han sido reformuladas, en aras del refuerzo del aprendizaje y la atención. De este modo, las presentaciones contienen la "información esencial" del temario, mientras que otra información secundaria como determinados ejemplos o aclaraciones adicionales no se incorpora de forma intencionada, dejándose huecos o espacios en blanco que el alumnado debe completar al hilo de las explicaciones. Con ello, se favorece la interacción, pues el alumnado debe estar pendiente y tomar anotaciones evitándose "los convidados de piedra", pues aquellas y aquellos alumnos que no estén pendientes o se despisten en otras tareas (whatsapp, redes sociales, mail u otras) estarán en desventaja con respecto al resto de compañeras y compañeros del grupo que sí ha prestado atención a la clase y se ha preocupado de completar el temario. Ello es especialmente importante en la situación actual, donde el acceso a bibliotecas y a manuales se encuentra limitado por la pandemia, y es esencial disponer de la mayor información posible para enfrentarse a las pruebas objetivas u exámenes. Por supuesto, durante las sesiones se han resuelto todas aquellas dudas y partes de la información que no se haya conseguido identificar, pues no se trata de que el alumnado se encuentre en una situación permanente de tensión, sino simplemente, que preste atención e interactúe con la clase, y si llegado el caso alguna alumna o alumno no ha podido hacer acopio de la información, se le ha facilitado a través de tutoría on line, siempre que hubiera asistido a la clase (presencial o virtualmente) o haya justificado no haber podido asistir.

Por otro lado, respecto del enfoque práctico de las asignaturas, más esencial que nunca por la coyuntura on line, ha sido potenciado. Ante la dificultad de realizar el tradicional caso práctico en el aula, en primer lugar, se ha optado por encargar algunos casos prácticos que el alumnado ha tenido que preparar previamente y que se han resuelto durante la clase, poniendo en común las soluciones. Y, en segundo lugar, se ha apostado por la gamificación del aprendizaje que consiste en la utilización de dinámicas propias del juego en contextos no lúdicos (Deterting et al., 2001:8), y que contribuyen a motivar al alumnado en su proceso cognitivo dada su predisposición natural positiva de este hacia el juego (Almonte \& Bravo, 2016:53-54). Por ello, se ha recurrido a la elaboración de kahoots que se realizan al final de cada tema, compartiendo pantalla en la aplicación informática. De este modo, el alumnado que está físicamente en el aula ve proyectadas las preguntas, mientras que el que sigue la clase desde casa puede realizarlo también, pues le aparece el kahoot en la pantalla compartida. En particular, es una herramienta especialmente útil para comprobar que los 
conocimientos se han asimilado y despejar las dudas, al tiempo que permite evaluar a aquel alumnado más tímido que por diversas razones no ha intervenido en las clases (bien sea presencial o virtualmente).

En cualquier caso, la realización de los kahoots no se ha configurado como una actividad obligatoria, pues depende de la estabilidad de la conexión de cada alumna o alumno el poder realizarlo correctamente (en ocasiones pueden producirse desconexiones sobrevenidas y no se puede penalizar al alumnado por esta circunstancia). Por ello, se ha apostado por una política de incentivo, su realización es voluntaria y el alumnado que obtenga las diez mejores puntuaciones sumará nota en la evaluación continua. Si bien, más allá de esta recompensa en la nota final del curso, lo importante es que tras cada pregunta se ha explicado cuál era la respuesta correcta y por qué, de forma que los contenidos del temario queden completamente aclarados, pues la utilización de aplicaciones o plataformas virtuales puede incitarles a implicarse más en el proceso de aprendizaje de la asignatura, puesto que están familiarizados con las mismas y estas forman parte de su cotidianeidad. Finalmente, este tipo de cuestionarios les ayuda a la hora de enfrentarse a las distintas pruebas puntuables de la asignatura (Moya \& Soler, 2018:1160).

\subsection{Derecho Internacional Público}

Las experiencias de innovación educativa que se presentan han sido desarrolladas en la asignatura Derecho Internacional Público, impartida en el tercer curso del Grado en Derecho en la Universidad de Alicante.

En primer lugar, se ha utilizado la plataforma educativa Quizizz, la cual permite la evaluación del alumnado a través de cuestionarios digitales. Este tipo de plataforma digital de gamificación es muy frecuente en la enseñanza universitaria y, en particular, en los estudios de Derecho, donde se combinan con la clásica lección magistral que continúa siendo la principal forma de transmisión de los conocimientos en el aula, tal y como se ha señalado en la Introducción (Martínez et al., 2018:685). En concreto, el docente ha creado un cuestionario compuesto de diez preguntas con cuatro alternativas de respuesta sobre cada una de las lecciones del temario. Con anterioridad a su realización en el aula (presencial o virtual) y en el plazo de una semana los estudiantes han debido proceder al estudio y análisis de los materiales bibliográficos, legislativos y jurisprudenciales recomendados. Transcurrido este tiempo, el profesorado ha iniciado la clase con una breve introducción y contextualización del tema objeto de análisis para, acto seguido, realizar el cuestionario virtual, al que el alumnado debía responder a partir de los conocimientos adquiridos con carácter previo. La actividad no ha sido puntuada con una nota concreta, pues su objetivo era servir como herramienta de autoevaluación al alumnado en la docencia no presencial. Con ello se suple, al menos en parte, la primera parte de las clases presenciales de la asignatura de Derecho internacional público consistente en realizar unas preguntas al alumnado respecto lo estudiado en la última clase y resolver dudas antes de pasar a la siguiente lección.

En segundo lugar, se ha hecho uso de la web institucional de Naciones Unidas (www.un.org) para la enseñanza de la asignatura. Por tanto, se trata de una metodología basada en el uso de fuentes primarias de aprendizaje, esto es, aquellas que contienen el Derecho directamente aplicable (García et al., 2008:212). Por un lado, se ha hecho uso de los vídeos oficiales ubicados en la página web para explicar los órganos de Naciones Unidas. En unos casos, antes de dar comienzo a la sesión y con carácter previo a la explicación del docente, se ha procedido al visionado del video para que el alumnado tenga una visión general del órgano u organismo objeto de estudio. En otros casos, una vez concluida su explicación, en la última parte de la sesión, se ha visionado el video 
explicativo, a modo de resumen final de las explicaciones previas del profesorado. Por otro lado, se ha usado la documentación oficial ubicada en la web de la ONU para el aprendizaje de su funcionamiento. Durante el transcurso de las explicaciones, el docente se apoya en la información contenida en estas páginas para completar la información dada. Una vez concluida la explicación teórica, el alumnado ha procedido a realizar una serie de prácticas, en las cuales debía de compaginar los conocimientos teóricos adquiridos a partir de las explicaciones del profesorado, junto con las habilidades adquiridas sobre búsqueda de información a través de la web institucional.

En tercer lugar, se ha hecho uso de la prensa como método de enseñanza-aprendizaje en la docencia del Derecho Internacional Público. En la docencia universitaria actual es imprescindible relacionar las enseñanzas impartidas en el aula con la realidad jurídica a la que se va a enfrentar el estudiante universitario finalizados sus estudios (Gutiérrez \& Carrión, 2010:50). Asimismo, potencia la adquisición de competencias como son la búsqueda y selección de información, el sentido crítico y la opinión reflexiva por parte del alumnado (Gutiérrez \& Tyner, 2012:34). En concreto, para introducir la utilización de la prensa como metodología docente durante las sesiones correspondientes a la materia de Derecho Internacional Público, el profesorado aplicó diversas dinámicas de trabajo. A continuación, se explican algunas de ellas:

i. Una de las actividades realizadas consiste en la presentación de una noticia de prensa relacionada con un tema específico del programa de la asignatura, en el que los medios de comunicación habían cometido un error a la hora de plasmar la problemática jurídica del asunto. En esta actividad, el profesorado divide a los discentes en salas virtuales de entre siete y ocho personas, quienes deben debatir durante un tiempo predeterminado cuál es el error que presenta la noticia reflejada en el proyector del aula. Transcurrido el tiempo concedido por el docente, por turnos, cada grupo expone en el aula virtual común el error que su grupo ha identificado y como deberían los medios de comunicación corregir la noticia con el fin de plasmar con mayor rigor jurídico el suceso acontecido. Después de la intervención de todos los grupos, el docente desvela cuál es el error clave en la noticia y repasa el contenido del punto del programa relacionado con la noticia de prensa objeto de debate.

ii. Otra de las actividades propuestas consiste en la presentación de una noticia de prensa de actualidad por el profesorado de la asignatura, de la que únicamente se daba detalle del titular y la información principal de la noticia. A raíz de estos datos, los discentes, por grupos (divididos en distintas salas virtuales) y en un tiempo predeterminado, debía identificar la problemática o el hecho jurídico derivado de la noticia y relacionarlo con un punto del temario explicado, motivando la su respuesta.

iii. El alumnado debía dividirse en grupos. Cada grupo debía escoger un punto del temario sobre el que hacer una exposición frente al resto de sus compañeros, en la sala virtual común. La exposición consiste en explicar el contenido de ese punto concreto y ponerlo en relación con un suceso reciente que guarde relación con el tema expuesto. Seguidamente se inicia un debate guiado por el profesor en el que el resto de los estudiantes exponen su punto de vista con referencia a la noticia explicada por el grupo encargado de exponer el tema. Por ejemplo, un grupo debía explicar el punto referente a la Corte Penal Internacional, correspondiente a la lección 20 del temario. Para exponer el contenido de este Tribunal Penal Internacional, el grupo encargado de exponer esta materia utilizó la siguiente nota de prensa: El País, "El tribunal de la Haya eleva a cadena perpetua la sentencia a Radovan Karadžić". 


\subsection{Cooperación Jurídica Internacional}

La mayoría de experiencias descritas por el profesorado que participa en esta publicación refleja su docencia dentro del sistema dual, con un número minoritario de estudiantes presencial y otro amplio a distancia. La asignatura de Cooperación Jurídica Internacional se imparte en el segundo curso del Grado en Relaciones Internacionales de la UA y ha sido abordada de forma exclusivamente on line. Si se compara con el sistema mixto o dual, se entiende que impartir el curso de forma on line ha sido una circunstancia favorable, puesto que ha permitido a docente y discentes centrar los esfuerzos en adaptar el proceso de aprendizaje por medio de un único canal de comunicación, el no presencial. En primer lugar, ha sido fundamental realizar diversos cursos de formación para dominar técnicas de oratoria y aprendizaje que permitieran la docencia on line y herramientas digitales para realizar actividades formativas on line como la gamificación (dentro del Programa de Formación docente gestionado por el Instituto de Ciencias de la Educación de la Universidad de Alicante, ICE). En segundo lugar, se ha analizado cómo incorporar al formato a distancia metodologías docentes presenciales como el aprendizaje basado en problemas (Font, 2008:230) o la gamificación (López, 2015). Por último, el autoaprendizaje se ha encaminado al aprovechamiento de las funcionalidades de Moodle y las herramientas de Google para la docencia (gracias a los materiales recogidos por la web de la UA).

Para la docencia de la asignatura, se han empleado dos plataformas distintas, una para las sesiones eminentemente teóricas (herramienta Dual de UACloud) y otra para las prácticas (Google Classroom). En la plataforma Dual del UACloud se explicaban los contenidos en streaming, intentado fomentar la interacción con el alumnado, por medio de cuestiones relacionadas con la materia -repasando conocimientos adquiridos- o refiriendo ejemplos (cotidianos o sobre famosos). En particular, la utilización de la prensa resulta especialmente útil para la formación jurídica (Ogilvy, 1996:55). Cuando se trataba de preguntas con respuestas breves era preferible la participación por medio del chat, mientras que, si eran necesarias otras respuestas más elaboradas, se solicitaba al alumnado que conectase el micrófono para contestar de forma oral. Si resultaba interesante conocer la opinión de todo el grupo sobre una cuestión, se empleaba una encuesta inmediata, una funcionalidad que permite la plataforma Dual, de forma que cuando el alumnado responde, automáticamente aparecen los resultados porcentuales de las respuestas. Gracias a este recurso, por un lado, se obtenía un feedback de los "asistentes" y, por otro, era posible detectar si había usuarios conectados que no seguían las explicaciones. Para la autoevaluación se han utilizado cuestionarios de UACloud y otros instrumentos como Quizizz o Kahoot cuyo uso estaba orientado a fomentar la motivación del alumnado (Santana \& García, 2018:158-160).

Las sesiones prácticas se han orientado con herramientas de Google, en particular la plataforma de Google Meet para trabajar las prácticas individuales o por grupos y se requería al estudiante para que conectara la cámara para proponer la corrección de las prácticas, de forma que su exposición se pudiera seguir con una imagen además del sonido. Los supuestos prácticos más complejos se entregaban utilizando la aplicación Evaluación y Entrega de Práctica del UACloud. La utilización de dos plataformas ha permitido, por una parte, aprovechar las posibilidades técnicas de las que dispone cada una de estas plataformas, por otra parte, ha sido obligado determinar con antelación si la próxima sesión iba a ser teórica o práctica para avisar al alumnado de la plataforma a utilizar. Este último aspecto ha planteado el inconveniente de que algunos estudiantes no recordaban el tipo de sesión y debían cambiar de herramienta a la hora de comenzar la clase. Los contenidos docentes se han abordado por medio de lecciones intercaladas con cuestiones teórico- 
prácticas depositadas en Moodle. Esta plataforma ha resultado más conveniente para este fin que otras anteriormente utilizadas -como los materiales docentes del UACloud- dado que Moodle permite ordenar de forma muy gráfica gran variedad de recursos, páginas web $\mathrm{y}$ actividades agrupadas por lecciones.

Por último, la utilización de bases de datos y recursos electrónicos ha sido fundamental para el seguimiento de las sesiones o la realización de las prácticas. Especialmente relevante se considera el Atlas Judicial Europeo en materia civil, dentro del Portal Europeo de e-Justicia, la web de la Conferencia del La Haya de Derecho internacional privado o el Prontuario, que se han aprendido a manejar durante las sesiones y resultan imprescindibles para realizar las actividades propuestas. El uso de estos recursos no se plantea como un mero acceso a la información, sino que se propone aprender a navegar con criterio para aplicar los conocimientos a un caso concreto (Maestre, 2019:152). Esta metodología ha permitido reforzar la adquisición de competencias para el uso de herramientas digitales, mientras que cuando las clases eran presenciales se planteaba el inconveniente de utilizar aulas con suficientes equipos informáticos o que algunos y algunas estudiantes no disponían de un portátil durante las sesiones, por lo que, en este sentido, la adaptación al medio on line ha resultado de gran utilidad.

En cuanto a los criterios de evaluación de la asignatura, a grandes rasgos se mantienen los mismos que cuando la docencia era presencial. El uso de medios digitales permite disponer de un registro completo de los ejercicios, pruebas y exámenes, evitando la utilización de papel y los posibles problemas que se pueden dar si se extraviaran, tratándose de un sistema más eficiente que el de soporte físico. Sin embargo, con la evaluación on line existe un mayor riesgo de que se planteen conductas poco éticas, como recibir apoyo externo para contestar exámenes. A este respecto, se han tenido que abandonar las pruebas escritas de texto libre, que eran habituales de forma presencial, y se han propuesto cuestionarios tipo test. Se han utilizado Cuestionarios del UACloud y de Moodle, resultando esta segunda plataforma mucho más efectiva a la hora de evitar comportamientos fraudulentos para realizar los exámenes, fundamentalmente la opción de cuestiones aleatorias dentro de un banco de preguntas.

\subsection{Nacionalidad y Extranjería}

En la asignatura de Derecho de la Nacionalidad y la Extranjería, impartida en el cuarto curso del Grado en Relaciones Laborales y Recursos Humanos de la UMH, el cambio metodológico impuesto por la situación sanitaria se dirigió a las siguientes cuestiones: 1) modificar el sistema de impartición de docencia, al tener que adaptarlas a las sesiones duales y reforzar los contenidos integrados al formato virtual; 2) cambios en el sistema de evaluación de la asignatura, para pasar de un examen escrito y presencial, a un sistema de evaluación a distancia; y 3) adaptar la ejecución del programa de la asignatura para acercar a los estudiantes de una forma creativa a los casos reales relacionados con las situaciones que se abordan en el programa de la asignatura. Todo ello ha permitido revertir el reto que se planteaba, en una oportunidad para diversificar la docencia y, sobre todo, en el modo de generar habilidades en el estudiantado y poder evaluar el cumplimiento de los objetivos docentes.

Como punto de partida para la adaptación del sistema, cabe señalar que la UMH implementó medidas tecnológicas para que las sesiones virtuales fueran desarrolladas a través de la plataforma Google Meet, insertada en el perfil docente de cada una de las asignaturas, y se perfeccionaron las aplicaciones del campus virtual, a fin de utilizar las herramientas Materiales y Tareas de forma fácil y sencilla. Al hilo de ello, se promovieron acciones de formación a distancia para el profesorado y se perfeccionaron los mecanismos 
de desarrollo de los blogs de las asignaturas, que han adquirido un valor extraordinario, ya que constituyen un vehículo clave de comunicación alumnos-profesores en este tipo de enseñanza.

A partir de ahí, se generaron contenidos audiovisuales, materiales y esquemas de estudio para cada uno de los temas del programa de la asignatura y se grabaron las sesiones docentes a través de Google Meet. Todo ello se puso a disposición del estudiantado, en el blog de la asignatura. Además, el alumnado contaba con dos manuales de referencia, elaborados por los profesores del área. Se complementaron los materiales con sentencias, artículos jurídicos relativos a los temas del programa, noticias de actualidad y casos prácticos extraídos de la realidad jurídica para su solución por parte de los estudiantes. La elección de los supuestos basados en casos reales ha sido efectiva para alejarse de la tradicional visión teórica y "teoricista” del Derecho y motivar a los discentes en el proceso docente de aprendizaje (Paniza et al., 2016). A través de su solución, se ofrecía espacio de debate a los estudiantes acerca de las cuestiones más importantes de los temas del programa de estudio. Posteriormente, se daba paso a la valoración de los datos ofrecidos con respecto a una situación relativa al Derecho de la Nacionalidad (adquisición, pérdida o recuperación) o del Derecho de Extranjería (contrataciones en origen, regularización de extranjeros, o llegada de estudiantes internacionales, etc.) y a continuación se les dejaba un plazo de tiempo para la búsqueda de soluciones extraídas de la práctica a los casos de cada tema. Esto es, soluciones que por analogía pueden encontrarse en los diferentes tipos de resoluciones (judiciales o no) emitidas por las autoridades españolas o europeas, respecto a otros casos reales de igual calificación jurídica. Como puede verse, las sesiones teóricoprácticas duales se diseñaron para involucrar a los estudiantes en la búsqueda de respuestas judiciales y administrativas a los casos prácticos planteados en clases. También se utilizaron técnicas de juego para favorecer el aprendizaje, como intercambio de roles, cuestionarios de Kahoot, etc.

Asimismo, como parte de los proyectos de innovación docente, se desarrolló una sesión de cine, relativo a un tema actual del Derecho de Extranjería como el de los menores no acompañados, a través del visionado y posterior debate de la película "El viaje de Said". Tras la proyección del cortometraje se llevó a cabo un debate y la realización de un cuestionario y dos casos prácticos por parte de los estudiantes. A ello hay que añadir la grabación de programas de radio mensuales en Radio UMH. Las sesiones iniciales fueron dedicadas a temas de esta rama jurídica, tratados bajo el título "Casos y cosas de Derecho internacional privado" y llevados a cabo por los profesores del área de conocimiento contando con expertos y profesionales de la práctica como invitados.

De igual manera, a petición de los estudiantes se desarrollaron sesiones extras con la finalidad de abordar cuestiones del programa que resultaron de su interés y sobre las cuales quisieran profundizar.

Teniendo en cuenta que la guía docente de la asignatura incluye el sistema de Evaluación Continua como forma de evaluación junto al examen final -previsto para quienes no puedan acceder a la Evaluación Continua o no la superan- ello permitió adaptar el sistema a partir de la realización de tres pruebas parciales de Evaluación a Distancia. Dichas pruebas, de carácter eminentemente práctico, iban dirigidas a fomentar el estudio individual y el análisis crítico por parte de los estudiantes. Las prácticas fueron compartidas a través del apartado de Tareas del Campus Virtual y debían ser entregadas por el alumnado por la misma vía. Una vez cerrado el plazo de entrega, se procedió a la corrección colectiva de las mismas, con la finalidad de que los estudiantes conocieran las respuestas correctas para cada caso y pudieran realizar autoevaluación de sus resultados, desde un punto de vista cualitativo, ya que la parte cuantitativa estaba dirigida a obtener el aprobado en 2 de 
las 3 pruebas para -en conjunto con la participación activa y la entrega de los supuestos prácticos semanales- aprobar la asignatura de manera exitosa. Fue muy significativo que todos los estudiantes que al inicio de curso eligieron la Evaluación Continua se mantuvieran en ella y que se motivaran con la asignatura de forma tal que hasta solicitaron sesiones extras de docencia. Cabe señalar que el $100 \%$ de los estudiantes inscritos en la Evaluación Continua superó la asignatura por esta vía.

El examen final, para quienes no formaron parte de la Evaluación Continua, se desarrolló de forma presencial a partir de preguntas teórico-prácticas respondidas por el alumnado con la particularidad de que pudieron utilizar los materiales de apoyo para el examen (normativa, esquemas, etc.) ya que no son preguntas de reproducción memorística, si no de razonamiento y de tipo práctico.

\subsection{Derecho Penal Especial}

La guía docente de esta asignatura se imparte en el tercer curso del Grado en Criminología de la UA y exige que se oferte el sistema de evaluación continua, extremo que requiere la participación activa del alumnado. Así las cosas, se ha optado por el modelo pedagógico flipped classroom (Jiménez, 2019), en el que los alumnos trabajan en grupos de cinco y se les asigna una de las lecciones del temario, así como una serie de recursos didácticos y materiales para que puedan estudiar los contenidos (materiales audiovisuales, bibliografía, artículos específicos, etc.).

El alumnado debía sintetizar los aspectos jurídicos más relevantes de un determinado delito, el que la docente asignara al grupo. Los objetivos didácticos que se pretendían conseguir con esta metodología eran: (i) aplicar los contenidos de la asignatura a los casos prácticos propuestos; (ii) organizar y repartir las tareas entre los miembros del grupo (autogestión y co-responsabilización); (iii) identificar y aislar la problemática y aspectos jurídicos relevantes del delito asignado; y, finalmente, (iv) sintetizar y exponer en 40 minutos los contenidos (bien jurídico protegido, objeto material, sujetos activos y pasivos, conductas típicas, problemas de interpretación y concursales).

Las herramientas tecnológicas empleadas a tal fin, han sido las que siguen:

- Herramientas de comunicación: Aula Virtual, Grupos de trabajo, tutorías virtuales, foros de dudas.

- Herramientas para la creación colaborativa: Nearpod

- Herramientas creación de grupos: Aula Virtual.

- Herramientas para la gestión de recursos: Entrega de prácticas.

- Herramientas evaluación colaborativa: Corubrics.

El rol de la docente consistió en asignar los temas objeto de estudio, facilitar el material y los recursos para la confección del tema, resolver dudas y revisar los trabajos grupales antes de que se expusieran al resto de alumnos/as, integrar las actividades propuestas por los grupos en la plataforma Nearpod y moderar los debates y las preguntas (Jiménez et al., 2020).

La tarea del alumnado consistió en constituir los grupos de cinco personas, a los que se asignó un número que debían incluir en las entregas de los trabajos. Con el material y recursos facilitados por la docente y los que ellos/as tuvieron a bien incorporar, elaboraron el tema que les había sido asignado. Al resto del alumnado se le facilitaba y explicaba la rúbrica con los ítems que debían emplear para evaluar a los miembros del grupo que estaban 
exponiendo. Cada grupo debía concertar, al menos, una vídeo tutoría por Google Meet o aula virtual de UACloud para resolver dudas que generadas por el estudio del tema y para que la profesora revisara los contenidos que iban a exponer. Los/as alumnos/as optaron por elaborar presentaciones con el programa Prezi y por realizar vídeos con la grabación de la explicación sincronizada con la presentación que proyectaban a través de YouTube. Tanto la exposición del tema, cuanto la aclaración de dudas y realización de las tareas, se realizó a través del Aula dual en UACloud, en sesión síncrona.

Cada grupo entregaba: la lección desarrollada, un esquema de la lección, cinco preguntas test y dos preguntas de debate o actividades feedback para el resto de compañeros/as, así como el enlace al vídeo de YouTube, a través de la herramienta Entrega de prácticas de UACloud. La docente, una vez realizada dicha entrega, gamificaba las preguntas test propuestas por el grupo y las actividades en Nearpod.

Después de que todos los grupos hubieran expuesto, obtenían un esquema-resumen de cada tema, así como cinco preguntas test que debían realizar ellos/as mismos/as, lo que les permitió la asimilación de contenidos y la posibilidad de "entrenar" con las preguntas test y les redujo la ansiedad para afrontar los exámenes test.

Tras el estudio de cada tema, se planteaba al alumnado una batería de casos reales, de los que se extraían los hechos probados de los escritos de acusación para que los/as alumnos/as tipificaran los delitos, aislaran problemas jurídicos y aplicaran los conocimientos adquiridos. Los casos se resolvieron en grupos, se entregaron a través de la herramienta Controles de UACloud y se expusieron de manera síncrona en clase.

\subsection{Historia del Derecho y de las Instituciones}

Historia del Derecho Español es una asignatura de formación básica y de carácter obligatorio que se cursa durante el primer año académico del Grado en Derecho. Esta asignatura otorga una base de conocimientos previos sobre cómo se origina, organiza y desarrolla el sistema jurídico español, sin la cual resultaría difícil comprender muchas de las restantes asignaturas del Grado (Sainz, 2008; 28-32). De ahí su importancia y la necesidad de una impartición adecuada de la materia, que siente las bases para el estudio de futuros contenidos del ámbito jurídico.

En este sentido, tras decretarse el pasado año el estado de alarma por la epidemia de COVID-19, la dirección de la asignatura, como otras muchas del Grado, se vio obligada a adaptar o adecuar el plan de estudio y sistema de evaluación a las nuevas y extraordinarias circunstancias derivadas de la emergencia sanitaria. Para ello, se trasladó la impartición del contenido teórico a la herramienta de Docencia Dual de UACloud, la cual se empleaba para impartir una clase magistral expositiva en el entorno virtual. No obstante, también se realizaron ciertas actividades innovadoras con el alumnado para facilitar su adaptación y la transición hacia esta nueva modalidad. Entre ellas, destaca el denominado "TrabajoPARES".

En virtud de dicha actividad, el alumnado de la asignatura ha realizado un trabajo evaluable consistente en la búsqueda de documentación jurídica y legislación histórica a través del Portal de Archivos Españoles (en adelante, "PARES"). Se trata de la principal plataforma de divulgación del Patrimonio Histórico Documental Español, que ha sido creada y desarrollada por la Subdirección General de los Archivos Estatales, del Ministerio de Cultura y Deporte. PARES alberga millares de fichas descriptivas de los fondos documentales conservados en distintos archivos históricos, entre otros, del Archivo Histórico Nacional y Archivo General de Simancas. Mediante el manejo (gratuito) de esta extraordinaria herramienta de investigación, el alumnado de Historia del Derecho puede 
realizar todo tipo de búsqueda multilingües en las distintas lenguas oficiales del Estado español y acceder a la información (descriptiva y facsímil digital) y los repositorios de jurisprudencia, documentación de geografía histórica, economía y derecho, mapas, planos e imágenes digitales (Álvarez-Coca, 2010:221-222; Sánchez, 2011: 73-82).

Tras una explicación práctica sobre el desarrollo de la actividad por el profesorado, el alumnado debía acceder desde su propia casa y de manera independiente al portal, para buscar y consultar todo tipo de documentación histórica desde la Edad Media hasta siglo XX. La documentación debía estar relacionada con un tema en concreto, distinto para cada curso académico. El tema escogido para el curso 2020/2021 fue "La problemática sanitaria en la historia de España”. Una vez consultado el catálogo de información disponible, así como la bibliografía recomendada por el profesor, cada grupo de trabajo -de dos integrantes como máximo- debía redactar un trabajo bien fundamentado y argumentado, en donde se reflexionara y se analizaran distintas cuestiones jurídicas al hilo de su estudio. La extensión máxima del trabajo era de 10 folios y debía constar de las siguientes partes: introducción, desarrollo, conclusión y fuentes. Los trabajos fueron remitidos al profesor responsable de la asignatura por tutoría, junto con un PowerPoint o video de presentación, a elección del alumnado participante. En ellos, se explicaban o narraban los objetivos del trabajo de manera concisa (máx. 6 minutos), así como la metodología empleada para su desarrollo y los principales resultados obtenidos a la luz de las fuentes legales y documentales consultadas.

Para la evaluación de la actividad PARES se tomaron en cuenta una serie de factores tales como la capacidad para buscar y analizar documentación en el portal de PARES, la justificación de la temática elegida en su evolución histórico-jurídica o la claridad y estructura apropiada tanto en la expresión escrita como oral.

La búsqueda de documentación histórica y su estudio y análisis pormenorizado ha permitido al alumnado formular opiniones y reflexiones jurídicas al hilo de sus investigaciones en el ámbito académico. Además, la elaboración del trabajo PARES también ha contribuido a facilitar la compresión -mediante la práctica- de numerosas cuestiones o aspectos jurídicos que le eran completamente desconocidos hasta la fecha.

\subsection{Derecho Procesal}

La asignatura de Derecho Procesal se imparte, entre otros, en el plan de estudios del Grado en Derecho y Administración de Empresa de la UA. Se trata de una asignatura subdividida en dos bloques totalmente diferenciados en contenido y en evaluación. En concreto, la experiencia que a continuación se narra se enmarca en la primera parte del programa correspondiente al "proceso civil".

De manera habitual, en esta asignatura se estudia un manual de referencia cuyo contenido íntegro constituye materia de examen. Por ello, las clases son magistrales expositivas y van acompañadas de ejemplos prácticos sencillos y expuestos sin mayores complicaciones en un entorno presencial. Con el cambio al aula virtual, se ha mantenido la clase magistral, si bien acompañada de diapositivas y reduciendo el temario de la asignatura. A su vez, la sesión teórica se ha reforzado con una parte práctica ampliada, la cual ha sufrido mayores cambios y se ha adaptado por completo al contexto digital.

De forma más particular, para el desarrollo de la parte práctica de la asignatura, se proporcionaba al estudiantado una serie de materiales de consulta, facilitándolos con una antelación mínima de 72 horas para que pudieran analizarlos debidamente (Arrabal, 2017:279). Entre estos documentos, se encontraban temas desarrollados en formato Word o PowerPoint, que en ocasiones contenían huecos a completar por las y los estudiantes, a 
modo de repaso y como una forma de incentivar el seguimiento activo de la lección. También se incluían videos de juicios y vistas reales para su debate en clase. Asimismo, se hizo uso de otros recursos más innovadores, como el visionado de videos ubicados en repositorios universitarios o accesibles desde redes sociales o canales de YouTube. Estos vídeos empleaban un registro más informal pero siempre haciendo referencia a contenido relacionado con la asignatura, como podrían ser explicaciones de la materia, juicios simulados o píldoras informativas. En este último caso, se les remitían los links o páginas web de consulta para su visionado y posterior comentario. Además, también se puso en práctica el análisis de la información vertida en medios digitales o audiovisuales (Twitter, prensa digital, etc.) por órganos oficiales y entidades públicas, que guardara relación con la materia. El uso de este tipo de recursos permitía aumentar la motivación de los estudiantes, lo cual es fundamental en materias que pueden resultar menos atractivas (Hernández-Ramos et al., 2021:4).

Otra de las experiencias docentes puesta en práctica durante este curso fue el seguimiento de un foro de discusión a través de la herramienta digital de Debates inserta en UACloud. En este foro, se permitía el planteamiento de dudas, que debían ser resueltas por los propios compañeros y compañeras, siempre bajo la supervisión de la docente responsable de la asignatura. También se fomentaba la referencia a material bibliográfico o didáctico que quisieran compartir con el resto para complementar el estudio de aquellos temas que desearan ampliar.

Por último, las tutorías presenciales se redujeron a las mínimas imprescindibles. Se estableció como método alternativo la videotutoría, aunque el alumnado, de manera general, optó por realizar las tutorías de la forma más tradicional, es decir, mediante mensajes escritos a través del correo electrónico o UACloud. Aun así, y a colación del párrafo anterior, se dispuso otro foro similar para plantear cuestiones de índole administrativa (fechas de examen, faltas, justificantes, guía docente, etc.).

En cuanto a la evaluación de la asignatura, también se ha visto afectada y ha sufrido modificaciones con el objetivo de adaptarla al sistema e-learning, dada la imposibilidad de convocar exámenes presenciales con cupos de cerca de cien estudiantes de media por grupo. Téngase en cuenta que, según las directrices establecidas en el departamento responsable de esta asignatura, las pruebas de evaluación se realizan simultáneamente a todos los grupos existentes en cada titulación. En este sentido, se optó por configurar una prueba tipo test on line gestionada a través de la herramienta Cuestionarios incluida en UACloud. De igual manera, se ofrecía al estudiantado un canal de comunicación mediante Google Meet, creándose una reunión en cada grupo con conexión directa con el profesorado para resolver las dudas e incidencias surgidas en el desarrollo de la prueba. Este canal se constituía también como una vía para la comprobación de la identidad de aquellos alumnos y alumnas cuya conexión se realizaba a través de una cuenta de correo electrónico no institucional.

\subsection{Derecho internacional privado}

La asignatura de la cual se hablará a continuación es la de Derecho internacional privado, impartida en el tercer curso del Grado en Derecho de la UMH. Con la llegada de la pandemia, la metodología docente teórico-práctica de la asignatura pasó a ser on line y se basó en la puesta a disposición del alumnado de diferentes contenidos docentes en el Campus virtual de la UMH (Universite). Para empezar, se publicaron sesiones docentes previamente grabadas por el profesorado ex profeso -siendo un total de 25 los videos grabados, de una duración media de 60-75 minutos cada uno- y se realizaron sesiones en directo, con la participación del estudiantado, a través de la herramienta Google Meet. Tales 
sesiones se llevaron a cabo en las franjas establecidas para la asignatura en los horarios oficiales de la titulación.

La docencia teórico-práctica se acompañó también de diferentes materiales escritos de carácter explicativo, elaborados por el profesorado del Área de Derecho internacional privado de la UMH al efecto. Entre ellos, se incluían casos prácticos, test de autoevaluación y otros materiales complementarios relativos a cada tema o materia perteneciente al contenido de la asignatura. Las prácticas remitidas debían realizarse y enviarse a través del e-mail y/o de formularios de Google y kahoots elaborados al efecto, por parte del alumnado durante un período de tiempo determinado. Ello permitía al profesorado del área evaluar adecuadamente la adquisición de los conocimientos y contenidos de la correspondiente materia por parte del estudiantado (Colina, 2017: 374). Asimismo, también se ofrecía semanalmente al alumnado un material denominado "Lecturas de Derecho internacional privado para el confinamiento", con artículos doctrinales o comentarios de sentencias relacionados con las 19 lecciones del programa de la asignatura para ampliar su contenido.

La tutorización de los estudiantes fuera del horario de clase se realizó a través de correo electrónico, respondiendo a las dudas planteadas por el alumnado, o bien mediante la herramienta Google Meet, en horario previamente anunciado por el profesorado.

El sistema de evaluación de la asignatura siguió partiendo de la valoración de los contenidos teóricos y las actividades prácticas realizadas por el alumnado y se llevó a cabo a través de un "sistema de evaluación continua a distancia" o mediante un "examen final no presencial":

En el "sistema de evaluación continua a distancia" se tuvo en cuenta la resolución de casos prácticos, test de autoevaluación y la realización de trabajos, reflexiones escritas y/o comentarios jurisprudenciales a lo largo del semestre de docencia. Ahora bien, la evaluación final de la asignatura en el "sistema de evaluación continua a distancia" consistió, en particular, en la resolución por escrito de 3 pruebas teórico-prácticas que se pusieron a disposición del alumnado en el Campus Virtual de la UMH (mediante Anuncios de la asignatura o Tareas), con una antelación suficiente, durante un periodo de aproximadamente un mes. El alumno que obtuvo una calificación igual o superior a 5 puntos superó la asignatura.

La evaluación de la asignatura a través de un "examen final no presencial" consistió en la realización de un examen final teórico-práctico oral para aquellos/as alumnos/as que no siguieron/hubieron superado la evaluación continua a distancia, a través de la herramienta Google Meet, en la que se valoró el grado de adquisición de los conocimientos y competencias por parte del alumnado y su aplicación práctica en la resolución de supuestos concretos.

\subsection{Derecho del Trabajo}

La asignatura de Derecho del Trabajo I, perteneciente al tercer curso del Grado en Derecho, se ha impartido conforme al régimen dual establecido por la UA. La clase estaba conformada por 113 alumnos y el seguimiento fue en su mayoría de manera telemática, aunque en todo momento hubo alumnos que acudieron presencialmente.

A la hora de plantear la estrategia docente de la asignatura se han tenido en cuenta dos condicionantes fundamentales: el carácter dual de la docencia y la provisionalidad en la que se la que se desenvuelve la actividad. En primer lugar, la docencia dual implica la asistencia presencial y telemática del alumnado de manera simultánea, lo que ha determinado que todas las técnicas y materiales empleados tuvieran que adaptarse de 
manera óptima a ambos entornos. El otro gran condicionante a la hora de planificar la estrategia docente ha sido la provisionalidad a la que estaba sometida la actividad, puesto que, ante un empeoramiento de la situación sanitaria, en absoluto descartable, se podía pasar a la docencia y evaluación exclusivamente on line, como finalmente aconteció. Por ello, todas la actividades y técnicas empleadas tenían que ser susceptibles de aplicación en cualquier entorno (presencial o virtual).

En esta línea de trabajo, las clases se han impartido utilizando la plataforma Docencia Dual disponible en UACloud. Como se ha expuesto anteriormente, esta aplicación permite retransmitir la clase impartida en el aula en streaming, de modo que los alumnos on line podían seguir las explicaciones en directo. Para facilitar el seguimiento de la lección, se ha optado por impartir las clases utilizando presentaciones PowerPoint como soporte visual, en las que se preveían anticipadamente los posibles ejemplos y esquemas necesarios para aderezar las explicaciones. Las presentaciones tienen la indudable ventaja para los alumnos de ser utilizadas como esquemas para preparar la materia.

En cuanto a la dinámica para el desarrollo de la clase, uno de los objetivos prioritarios ha sido mantener la atención de los alumnos. En efecto, la docencia on line tiene como efecto pernicioso la dificultad para la mantener la atención en las explicaciones (LópezGonzález, 2020). El hecho de que los alumnos no tengan activada su cámara da lugar a que, en muchas ocasiones, sintiéndose libres de la supervisión por parte del profesor, desatiendan las explicaciones.

Para fomentar su atención, se aumentó el volumen de prácticas a realizar en clase y se fueron realizando a conveniencia, sin tener en cuenta si formalmente se estaba en una clase teórica o práctica, lo que les obligaba a estar más pendientes. Para realizar las prácticas se empleó la utilidad Salas Externas de Docencia Dual, que permitía crear instantáneamente grupos de trabajo en los que los alumnos podían debatir el tema en petit comité durante el tiempo que se les proporcionaba. Además, la plataforma permite al docente acceder a cada pequeño grupo de trabajo para resolver dudas y controlar que la actividad se esté realizando correctamente. Finalizado el tiempo, volvían automáticamente al aula para resolver en común el caso. Por otro lado, además de estar los alumnos más atentos a las clases, la proximidad de la explicación teórica y la realización de la práctica en la misma sesión favorecía mucho más su comprensión.

Los materiales empleados han sido los habituales: manual básico y complementarios, esquemas, presentaciones, etc. La única novedad es que para los materiales proporcionados se ha utilizado la plataforma Moodle, que permite organizar mucho mejor el contenido y, además, presenta utilidades que en UACloud están más limitadas.

La elección del sistema de evaluación y los criterios utilizados también se ha visto condicionada por la inevitable provisionalidad a la que estaba sometida la actividad docente (López, 2021:288-289). Por este motivo, el sistema de evaluación se planteó considerando dos escenarios: la evaluación presencial y la evaluación on line. Y teniendo en cuenta la inevitable vinculación entre las estrategias docentes y la evaluación de los aprendizajes, era preciso, además, que el sistema de evaluación empleado fuera el mismo independientemente del escenario en el que se tuviera que desarrollar.

La opción finalmente elegida fue la de realizar un examen oral, ya fuera de manera presencia u on line. Las razones por las que se prescindió de la modalidad escrita fueron dos. Por una parte, un examen tipo test para el Grado en Derecho no parece la forma más adecuada para valorar las capacidades del alumnado, puesto que no refleja destrezas esenciales como la capacidad de expresión o la capacidad de análisis y síntesis. Por otra parte, las dificultades para controlar la limpieza en el desarrollo de la prueba, dado que los 
alumnos no están obligados a encender las cámaras durante su desarrollo, también desaconsejan la utilización de la modalidad escrita en cualquiera de sus variantes (test o desarrollo). Así las cosas, se optó por la realización de exámenes orales, técnica susceptible de ser empleado tanto de manear presencial como on line. De este modo, el examen parcial fue presencial y los alumnos se examinaron de manera tradicional, esto es frente a un tribunal. Por supuesto se extremaron todas las medidas de precaución necesarias para garantizar la seguridad sanitaria en todo momento (citación en una hora concreta, salas grandes y ventiladas etc.) Sin embargo, el examen final, dado el recrudecimiento de la situación sanitaria, se realizó on line. Para su ejecución, la herramienta utilizada ha sido Moodle. La elección de esta plataforma responde a dos razones: su versatilidad y las garantías que ofrece para evitar el fraude en la realización del examen.

\section{Valoración de los resultados}

Para valorar los resultados de la adaptación de la docencia al entorno virtual, se ha realizado una encuesta al alumnado (un total de 138 estudiantes) compuesta por 5 preguntas acerca de las actividades y metodologías desplegadas. Asimismo, el profesorado participante (10 docentes) ha rellenado un cuestionario plasmando su experiencia durante el curso académico 2020/2021. Todo ello, con el objetivo último de determinar el grado general de satisfacción con el desarrollo de las clases on line y/o semipresenciales.

A continuación, se realiza un análisis cuantitativo de los datos obtenidos en cada una de las preguntas formuladas al muestreo no probabilístico causal de discentes (Bisquerra, 2009:148):

En primer lugar, a la pregunta de cuál era su opinión sobre la adaptación a la docencia on line en las asignaturas de Derecho, el 94,93\% del alumnado ha respondido de manera positiva, mostrándose satisfecho con la metodología empleada por el profesor responsable de la asignatura. De esta forma, únicamente un 5,07\% (7 estudiantes) ha considerado que la adaptación fue desfavorable.

\begin{tabular}{|c|c|c|}
\hline $\begin{array}{l}\text { Opinión docencic } \\
\text { on line }\end{array}$ & Absolutos & Porcentajes \\
\hline \multirow{4}{*}{$\begin{array}{l}\text { Favorable } \\
\text { Desfavorable } \\
\text { NS/NC } \\
\text { TOTAL }\end{array}$} & 131 & $94,93 \%$ \\
\hline & 7 & $5,07 \%$ \\
\hline & 0 & $0,00 \%$ \\
\hline & 138 & $100,00 \%$ \\
\hline
\end{tabular}

En segundo lugar, un $52,17 \%$ del alumnado encuestado ha coincidido en que su motivación para la realización de las actividades on line fue "mayor que las realizadas de forma presencial", mientras que un $14,49 \%$ ha considerado que fue "igual" y un 33,33\% ha señalado que fue "menor que las realizadas de forma presencial".

\begin{tabular}{|c|c|c|}
\hline Motivación & Absolutos & Porcentajes \\
\hline Igual & 20 & $14,49 \%$ \\
\hline Menor & 46 & $33,33 \%$ \\
\hline Mayor & 72 & $52,17 \%$ \\
\hline$N S / N C$ & 0 & $0,00 \%$ \\
\hline TOTAL & 138 & $100,00 \%$ \\
\hline
\end{tabular}


En esta misma línea, a la pregunta de si "¿Considera que con la docencia on line ha aprendido más, menos o igual que con la docencia presencial?", el 50\% de los encuestados ha manifestado haber aprendido "más que con la docencia presencial", mientras que un $31,88 \%$ ha considerado haber aprendido "menos que con la docencia presencial". El $16,67 \%$ restante ha afirmado haber aprendido "igual" y cabe señalar que un $1,45 \%$ de los encuestados ( 2 estudiantes) ha decidido no responder a la pregunta.

\begin{tabular}{|c|c|c|}
\hline Aprendizaje & Absolutos & Porcentajes \\
\hline \multirow{5}{*}{$\begin{array}{l}\text { Igual } \\
\text { Menor } \\
\text { Mayor } \\
\text { NS/NC } \\
\text { TOTAL }\end{array}$} & 23 & $16,67 \%$ \\
\hline & 44 & $31,88 \%$ \\
\hline & 69 & $50,00 \%$ \\
\hline & 2 & $1,45 \%$ \\
\hline & 138 & $100,00 \%$ \\
\hline
\end{tabular}

Entre todas las actividades realizadas durante el desarrollo de las clases, el 52,17\% del alumnado ha considerado que los "Cuestionarios (UACloud, Moodle, Kahoot, etc.)" fueron la herramienta más útil para su formación en las distintas asignaturas. En contraposición, el $28,99 \%$ de estudiantes se ha decantado por "las sesiones por el modelo Dual", mientras que un 15,54\% ha preferido la realización de "actividades prácticas (Google Classroom, Google Meet, entrega de prácticas)". Por último, un 2,9\% de los encuestados (4 estudiantes) ha decidido no contestar a esta cuestión.

\begin{tabular}{c|rr}
\multicolumn{1}{c}{$\begin{array}{c}\text { Actividades } \\
\text { más útiles }\end{array}$} & Absolutos & Porcentajes \\
\hline Cuestionarios & & \\
Actividades & 72 & $52,17 \%$ \\
prácticas & 22 & $15,94 \%$ \\
Sesiones Dual & & \\
NS/NC & $4 C$ & $28,99 \%$ \\
TOTAL & 4 & $2,90 \%$ \\
& $13 \varepsilon$ & $100,00 \%$
\end{tabular}

Finalmente, a la pregunta de si “¿Recomendaría la docencia on line?”, el 76,09\% de estudiantes (un total de 105 discentes) ha contestado que sí la recomendaría, frente a un $20,29 \%$ que se muestra en contra de este modelo y un 3,62\% que no tiene una opinión clara acerca del sistema de docencia on line y/o dual.

\begin{tabular}{c|ll}
$\begin{array}{c}\text { Recomienda } \\
\text { docencia } \text { on line }\end{array}$ & Absolutos & Porcentajes \\
\hline SÍ & 105 & $76,09 \%$ \\
NO & 28 & $20,29 \%$ \\
NS/NC & 5 & $3,62 \%$ \\
TOTAL & 138 & $100,00 \%$
\end{tabular}

En cuanto a la valoración de los resultados obtenidos por parte del profesorado, los cuestionarios realizados han servido para conocer su punto de vista y extraer ciertas conclusiones comunes sobre el desarrollo de las clases en el entorno virtual, así como para confirmar los datos obtenidos en las encuestas realizadas al alumnado. 
En este sentido, el 100\% de los docentes considera que los resultados obtenidos a partir de las estrategias docentes y las herramientas virtuales aquí expuestas han sido muy positivos. El nivel de adquisición de las competencias establecidas en las correspondientes guías docentes es igual, sino superior, al obtenido a través de la enseñanza presencial. Asimismo, se han alcanzado de manera suficiente los resultados de aprendizaje u objetivos formativos previstos para las distintas asignaturas. Los discentes que han participado de manera activa a lo largo de las sesiones han mostrado un buen nivel de comprensión de los contenidos impartidos, así como una adecuada puesta en práctica de los mismos. Ejemplo de ello es el porcentaje de estudiantes aprobados, el cual se mantiene en el mismo nivel que en cursos anteriores en los que la docencia era únicamente presencial.

No obstante, el equipo docente ha reflejado también una serie de dificultades al correcto desarrollo de las sesiones virtuales que conviene poner de manifiesto. Con carácter general, se trata de obstáculos de naturaleza interdisciplinar o transversal, es decir, comunes a todas las asignaturas expuestas pese a las diferencias de contenido existentes entre ellas. Además, la mayoría son problemas inherentes al entorno de docencia on line o semipresencial y sobre los cuales se debe seguir trabajando a efectos de ofrecer una mejor docencia al alumnado y enriquecer el proceso de enseñanza-aprendizaje.

En concreto, uno de los mayores inconvenientes planteados ha sido el de la falta de participación real por un sector del alumnado. El número de personas conectadas a las diferentes plataformas era notable y se correspondía al que cabría esperar de una clase presencial. No obstante, únicamente un número limitado de estudiantes ha participado de manera fehaciente. Además, en la mayoría de los casos el alumnado no hacía uso de las cámaras y micrófonos disponibles, sino que se interactuaba solo a través del chat. Ello dificultaba conocer si el resto de participantes prestaba atención a la clase o, por el contrario, se trataba de una presencia meramente anecdótica. La existencia de un mayor número de posibles distracciones fuera del contexto del aula presencial no ha contribuido a reducir dicha incertidumbre.

Otra de las dificultades observadas fue la necesidad de atender de manera simultánea al alumnado que acudía de manera presencial y al alumnado conectado a través de la plataforma on line en los grupos adheridos a los sistemas de docencia dual. El limitado campo de visión de las cámaras habilitadas en las aulas, junto con la baja potencia de los micrófonos, complicaba la atención de la clase para las y los estudiantes conectados on line. A todo ello, hay que sumar la complejidad de exponer la materia correspondiente a dos grupos distintos a la vez sin desatender las necesidades particulares de uno y de otro. Esta circunstancia condicionaba la dinámica de las clases y provocaba una acusada lentitud en la impartición de los contenidos.

Por último, deben destacarse también los problemas de conexión experimentados durante el desarrollo de las clases. En determinados momentos, el alumnado ha llegado a comunicar que la calidad de imagen y/o de sonido era deficiente debido a la débil conexión de red. Ello ha provocado interrupciones constantes y ha añadido un grado extra de dificultad a la explicación de los contenidos. Además, también cabe señalar las limitaciones de los dispositivos empleados por el estudiantado, quien no siempre disponía de la tecnología adecuada para el seguimiento de la clase.

El conjunto de obstáculos presentado evidencia que los sistemas de docencia on line y dual están lejos de la perfección. Tal y como señala el profesorado participante, es necesario realizar mejoras que permitan solventar los problemas aquí observados. Si bien no es el objeto principal de este trabajo, a continuación, se detallan algunas de las sugerencias realizadas por los docentes que podrían contribuir a perfeccionar el sistema de 
docencia virtual. La primera de ellas se basa en lograr una mayor participación activa del alumnado animándolo a hacer empleo de las herramientas tecnológicas disponibles (videocámaras y micrófonos). Ello permitiría, por un lado, comprobar su asistencia real y, por el otro, alcanzar un alto grado de interacción; lo que a su vez facilitaría la resolución de dudas y el intercambio de opiniones entre los participantes, más allá del cruce de mensajes por el chat, cuya funcionalidad puede ser un tanto limitada en el contexto del aula. La segunda de estas propuestas se basa en continuar apostando por el uso de métodos o herramientas como Kahoot, Nearpod o Quizizz, que sean capaces de atraer la atención del alumnado y contribuir a un acercamiento dinámico al contenido de la asignatura. En último lugar, si bien queda fuera de la esfera de control del equipo docente, se recomienda también la apuesta por la creación de planes y ayudas orientados a suplir las carencias observadas en el estudiantado en términos de acceso a Internet y a tecnologías adecuadas para el seguimiento de la clase. Ejemplo de ello son las Ayudas de Emergencia a Estudiantes UA pertenecientes al Plan de Continuidad aprobado por la Universidad de Alicante en mayo de $2020^{2}$.

Con todo, no debe obviarse la responsabilidad del alumnado en el proceso de enseñanza-aprendizaje. La existencia de mayores dificultades para el seguimiento de la asignatura por los discentes en el contexto pandémico es innegable y estas deben ser necesariamente tenidas en cuenta a la hora de desarrollar las distintas estrategias docentes. Ahora bien, en la medida de lo posible, los estudiantes deben procurar asumir un rol activo durante el desarrollo de la asignatura, que complemente los esfuerzos realizados por el profesor para contribuir a un aprovechamiento óptimo de los recursos y del tiempo dedicado (Hernández, 2008: 31-32; Falco, 2017: 62).

\section{Conclusiones}

Para concluir y con carácter general, puede parecer que la implementación de estrategias y metodologías docentes innovadoras al entorno on line en el ámbito de las ciencias jurídicas entraña una dificultad añadida, puesto que se trata de materia con grandes dosis de contenido teórico. Si bien, las estrategias y metodologías expuestas a lo largo de estas páginas dan buena prueba de lo contrario, es decir, demuestran que esta dificultad añadida es una mera ilusión.

El catálogo de herramientas y técnicas disponible para la adaptación a la docencia on line o semipresencial es lo suficientemente amplio. Todas las estrategias docentes e instrumentos referidos se han integrado de manera satisfactoria en el desarrollo de las clases. Asimismo, aplicaciones como Kahoot, Google Meet, YouTube o Nearpod han permitido involucrar al estudiantado de forma activa y lograr una adecuada comprensión de los contenidos por su parte. De esta manera, se ha garantizado una cierta continuidad en la impartición de las asignaturas pertenecientes al ámbito de las ciencias jurídicas.

Las principales dificultades observadas no van referidas al mal funcionamiento de estas herramientas. Tampoco a una respuesta negativa por parte del alumnado, quien ha recibido con entusiasmo el uso de dichas aplicaciones. Por el contrario, aquellos factores que han entorpecido la impartición de la asignatura se relacionan con variables externas que escapan al control del profesorado. Se trata de elementos tales como una deficiente conexión de red o la existencia de distracciones en el entorno familiar o personal de los estudiantes.

\footnotetext{
${ }^{2}$ Ver en: https://pdc.ua.es/es/alumnado.html, visitado el día 15 de septiembre de 2021.
} 
Los docentes han procurado salvar estos obstáculos y no dejar a ningún estudiante atrás, de acuerdo con el Objetivo n. 4 ínsito en los Objetivos de Desarrollo Sostenible de la Agenda 2030 de Naciones Unidas; esto es, la necesidad de garantizar una educación inclusiva, equitativa y de calidad, así como de promover oportunidades de aprendizaje para todo el mundo. Para ello, el profesorado ha adaptado las técnicas y herramientas empleadas cuando ha sido necesario: cambiando de plataforma virtual para el seguimiento de la clase, repitiendo la explicación si había errores de conexión, tomándose el tiempo necesario para ilustrar el uso de las herramientas o empleando metodologías activas para involucrar al alumnado y evitar posibles distracciones externas.

No obstante, este esfuerzo por parte del docente requiere de igual manera de un compromiso por parte del resto de miembros de la comunidad universitaria para asegurar el éxito del sistema de docencia virtual y semipresencial. Esto incluye tanto a estudiantes, quienes deben, dentro de sus posibilidades, consolidar su rol como participantes activos en el proceso de enseñanza-aprendizaje; así como a instituciones universitarias y órganos públicos, quienes deben contribuir a la solución de los problemas observados (acceso a Internet y tecnologías adecuadas, etc.) para facilitar la adaptación de la labor docente a un entorno on line y semipresencial. 


\section{Bibliografía}

- ALMONTE, M. G. \& BRAVO, J. (2016). Gamificación y e-learning: estudio de un contexto universitario para la adecuación de su diseño. Revista Tecnología, Ciencia y Educación, núm. 4, 52-60. https://doi.org/10.51302/tce.2016.78

- ÁlVAREZ-COCA GONZÁLEZ, M. J. (2010). La investigación histórica y los archivos en Internet. La presencia del Archivo Histórico Nacional en el Portal de Archivos Españoles (PARES). Cuadernos de Historia Moderna, núm. 35, 175-222. https://revistas.ucm.es/index.php/CHMO/article/view/CHMO1010110175A/21457

- ÁlvareZ-ROSA, C. V., VElasco, E., NEVOT, M., MARCET, V. M. \& CASTRILLO, I. (2018). La competencia digital en la universidad con la aplicación Kahoot. En REDINE (Ed.), Innovative strategies for Higher Education in Spain (pp. 49-57). Adaya Press.

- ARRABAL PLATERO, P. (2017). Innovación docente en los estudios semipresenciales. En Casanova Martí, V. T. (Ed.), Nuevos retos docentes en ciencias sociales y jurídicas (pp. 275-287). Huygens Editorial.

- BASTANTE GRANELL, V. \& MORENO GARCÍA, L. (2019). Plataforma digital «ludoteca jurídica»: una apuesta por la «gamificación» en Derecho. Innovación Educativa y Metodologías Docentes, núm. 21, 25-44. https://revistas.uma.es/index.php/rejienuevaepoca/article/view/7531

- BISQUERRA ALZINA, R. (Coord.) (2009). Metodología de la investigación educativa. Editorial La Muralla.

- COBO-RENDÓN, R., VEGA-VALENZUELA, A. \& GARCÍA-ÁLVAREZ, D. (2020). Consideraciones institucionales sobre la Salud Mental en estudiantes universitarios durante la pandemia de Covid-19. CienciAmérica, vol. 9, núm. extra 2, 277-284.

- COLINA VARGAS, A. (2017). Una mirada desde el pensamiento complejo a la apropiación de las TIC en la docencia universitaria. INNOVA Research Journal, vol. $2, \mathrm{~N}^{\mathrm{o}} 8,374-384$.

- DE CARVALHO GONÇALVES, S. M. (2017). Evaluation as a learning moment behavioural analysis in an e-learning environment [Tesis doctoral, Universidad de Vigo]. Investigo. http://www.investigo.biblioteca.uvigo.es/xmlui/bitstream/handle/11093/788/Evaluati on_as_a_learning_moment.pdf? sequence $=1 \&$ isAllowed $=\mathrm{y}$

- DETERTING, S., DIXON, D., KHALED, R. \& NACKE, L. E. (2011). Gamification. Toward a definition. Proceedings of the CHI 2011 Workshop Gamification: Using Game Design Elements in Non-Game Contexts, 6-9. http://gamificationresearch.org/wp-content/uploads/2011/04/02-Deterding-Khaled-Nacke-Dixon.pdf

- ElguETA ROSAS, M. F. \& PALMA GONZÁLEZ, E. E. (2014). Una propuesta de clasificación de la clase magistral impartida en la Facultad de Derecho. Revista Chilena de Derecho, vol. 41, núm. 3, 907-924. https://scielo.conicyt.cl/pdf/rchilder/v41n3/art06.pdf 
- FALCO, M. (2017). Reconsiderando las prácticas educativas: TICs en el proceso de enseñanza-aprendizaje. Tendencias pedagógicas, núm. 29, 59-76.

- FONT, A. (2008). El uso de las TIC como soporte para el ABP. En García Sevilla, J. (Coord.), El aprendizaje basado en problemas en la enseñanza universitaria (pp. 229253). Servicio de Publicaciones de la Universidad de Murcia.

- GARCÍA, J., GÓMEZ, T., ANDRÉS, F. J., ARANGÜENA, C., DE HOYOS, M. \& GUILLARTE, C. (2008). Reflexión sobre el papel de las TICs en la innovación metodológica de los estudios de Derecho. En Guilarte, C. (Coord.), Innovación docente: docencia y TICs (pp. 209-220). Universidad de Valladolid.

- GUTIÉRREZ, A. \& TYNER, K. (2012). Educación para los medios, alfabetización mediática y competencia digital. Comunicar. Revista Científica de Educomunicación, núm. 38, vol. XIX, 31-39.

- GUTIÉRREZ, M.V. \& CARRIÓN, D. (2010). Aplicación del dossier de prensa en la docencia universitaria. En Carrión, D. (Coord.), Claves docentes en el Espacio Europeo de Educación Superior: todo lo que necesitas saber para ser innovador y tener éxito en la docencia (pp. 45-58). Demiurgo Comunicación.

- HERNÁNDEZ-RAMOS, J. P., MARTÍNEZ-ABAD, F. \& SÁNCHEZ-PRIETO, J. C. (2021). El empleo de videotutoriales en la era post COVID19. Revista de Educación a Distancia, vol. 21, núm. 65. https://digitum.um.es/digitum/handle/10201/101144

- HERNÁNDEZ REQUENA, S. (2008). El modelo constructivista con las nuevas tecnologías: aplicado en el proceso de aprendizaje. Revista de Universidad y Sociedad del Conocimiento, vol. 5-2, 26-35. https://www.redalyc.org/pdf/780/78011201008.pdf

- JIMÉNEZ, D., GONZÁLEZ, J. J. \& TORNEL, M. (2020). Metodologías activas en la universidad y su relación con los enfoques de enseñanza. Profesorado, Revista de Currículum y Formación del Profesorado, 24(1), 76-94.

- JIMÉNEZ VAQUERIZO, E. (2019). Metodologías activas de aprendizaje en el aula: Apuesta por un cambio de paradigma educativo. Aula Magna McGraw Hill.

- LETELIER LOYOLA, E. (2017). Hacia el rescate del método socrático en el aprendizaje actual del derecho procesal. Opinión Jurídica, vol. 16, núm. 31, 117-134. http://www.scielo.org.co/pdf/ojum/v16n31/1692-2530-ojum-16-31-00117.pdf

- LÓPEZ GONZÁLEZ, J. L. (2020). Reflexiones sobre la experiencia docente semipresencial. Blog Facultad de Derecho. Recuperado el 15 de septiembre de 2021 de https://www.blog.fder.uam.es/2020/11/03/reflexiones-sobre-la-experienciadocente-semipresencial/

- LÓPEZ MARÍN, V. (2015). La Gamificación educativa. Una alternativa para la enseñanza creativa. Digital Education Review, núm. 27. https://revistes.ub.edu/index.php/der/article/view/13433/pdf

- LÓPEZ SÁNCHEZ, C. (2021). Ajustes sobrevenidos en la docencia a propósito de dos sistemas de evaluación on line. Cuadernos jurídicos del Instituto de Derecho Iberoamericano, núm. 1, 288-303.

- MAESTRE CASAS, P. (2019). La cooperación jurídica internacional en la docencia del grado en Derecho. En Cebrián Salvat, M.A., \& Lorente Martínez, I. (Dirs.). Innovación docente y Derecho internacional privado (pp. 139-158). Comares. 
- MARÉ, S. \& TEEDZWI, A. (2021). The effectiveness of etutoring in an open and distance e-learning environment: evidence from the university of South Africa. Open Learning: The Journal of Open, Distance and e-Learning, vol. 36(2), 164-180. https://doi.org/10.1080/02680513.2020.1717941

- MARTÍNEZ, P., PÉREZ, J. \& MARTÍNEZ, M. (2016). Las TICs y el entorno virtual para la tutoría universitaria. Educación XXI, 19(1), 287-310. https://doi.org/10.5944/educxx1.13942

- MARTÍNEZ MARTÍNEZ, N., BERENGUER ALBADALEJO, C., CABEDO SERNA, LL., EVANGELIO LLORCA, R., LÓPEZ RICHART, J. \& MÚRTULA LAFUENTE, V. (2018). La gamificación de la enseñanza del Derecho civil: la herramienta Quizizz. En Roig-Vila, Rosabel (Ed.), El compromiso académico y social a través de la investigación e innovación educativas en la Enseñanza Superior (pp. 684-693). Octaedro.

- MOYA FUENTES, M.D.M \& SOLER GARCÍA, C. (2018). La gamificación mediante herramientas virtuales de respuesta de audiencia. La experiencia de Socrative y Kahoot. En Roig-Vila, I. (Ed.), El compromiso académico y social a través de la investigación e innovación educativas en la Enseñanza Superior (pp. 1154-1163). Octaedro.

- MOYA-LACASA, C., ÁlVAREZ-VÁZQUEZ, C. M., GONZÁLEZ-BLANCO, L., VALTUEÑA-GARCÍA, M., MARTÍN-GIL, E., SEIJO-ZAZO, E., DE LA FUENTETOMÁS, L., GARCÍA-ÁlVAREZ, L., SÁIZ, P., GARCÍA-PORTILlA, M. \& BOBES, J. Impacto de la pandemia COVID-19 sobre la salud mental en las diferentes comunidades autónomas. Actas Españolas de Psiquiatría, vol. 49, núm. 2, 64-70.

- OGILVY, J.P. (1996). The use of journals in legal education. A tool for reflection, Clinical Law Review, vol. 3:55, 55-107.

- PANIZA, A., FERRER, B., AIGE M.B., VAGUER, F. \& MONSERRAT, M.I. (2016). La formación interdisciplinar de los futuros profesionales del Derecho a través de las TIC. En Roig-Vila R. (Coord.), Tecnología, innovación e investigación en los procesos de enseñanza-aprendizaje (pp. 2872-2881). Octaedro.

- SAINZ GUERRA, J. (2008). Historia del Derecho Español. Dykinson.

- SÁNCHEZ MAIRENA, A. (2011). El Portal de Archivos Españoles [PARES] como recurso para la historia local en internet. Canelobre: Revista del Instituto Alicantino de Cultura "Juan Gil-Albert", núm. 58, 73-82.

- SANTANA CABELLO, R. \& GARCÍA JUAN, L. (2018). La gamificación en la educación superior. En CIMTED (Ed.), Las Competencias y La Sociedad del Conocimiento (pp, 155-176). Editorial Corporación CIMTED.

- SOTO BERNABÉU, L. (2020). El Kahoot como método de Evaluación continua del aprendizaje en Derecho Financiero y Tributario. En Delgado, A.M., \& Beltrán, I. (Coord.), La docencia del Derecho en línea: cuando la innovación se convierte en necesidad (pp. 193-206). Huygens Editorial. 
- TRIPATHI, S. K. \& AMANN, W. C. (2020). COVID-19 y educación superior: Aprender a desaprender para crear una educación para el futuro. UNESCO. Recuperado el 15 de septiembre de 2021, de https://www.un.org/es/impactoacad\%C3\%A9mico/covid-19-y-educaci\%C3\%B3n-superior-aprender-desaprenderpara-crear-una-educaci\%C3\%B3n-para 